\title{
Utilidad de las películas para debatir temas complejos: política, religión y ciencia en Ágora
}

\author{
José Aramburu, Fèlix Bosch, Mariano Sentí, Josep E. Baños
}

Introducción. Las películas comerciales han constituido un método docente de demostrada eficacia en entornos educativos de ciencias de la salud. En el presente artículo se describe la utilidad de Ágora para presentar las complejas relaciones entre ciencia, política y religión, las cuales afectan a diversas situaciones biomédicas en la actualidad.

Materiales y métodos. La actividad consistió en la proyección de la película y posterior debate, al final del cual los estudiantes cumplimentaron, de forma anónima y voluntaria, un cuestionario de diez preguntas sobre los temas tratados y los vinculados con los objetivos educativos preestablecidos. Después se les solicitó que enviaran voluntariamente un informe personal sobre los aspectos más relevantes de la película.

Resultados. Cincuenta y dos estudiantes (96,3\% de los asistentes) respondieron el cuestionario de evaluación. Consideraron que Ágora tenía un interés notable para describir un ejemplo de conflicto entre ciencia y poderes sociales, y manifestaron que tales situaciones aún podían persistir hoy. En los informes personales realizados por 49 estudiantes (90,7\%) destacaron la consideración de tales conflictos, las barreras impuestas al conocimiento nuevo, la discriminación de la mujer en la sociedad y en la ciencia, así como las dificultades que entraña el respeto por el pensamiento individual.

Conclusiones. Ágora puede ser una película de interés para analizar y debatir las difíciles relaciones entre ciencia, religión y política. A pesar de su ambientación clásica, plantea situaciones aún identificables en nuestra sociedad y que los estudiantes deberían conocer.

Palabras clave. Ciencia. Heliocentrismo. Método científico. Películas comerciales. Política. Religión.

\section{Usefulness of movies for discussing complex topics: politics, religion and science in Ágora}

Introduction. Popular movies have turned out to be useful educational tools able to introduce complex issues to students of health sciences. The present paper describes the usefulness of Ágora to discuss the conflicts that might appear when science is faced with religion or politics.

Materials and methods. The movie was introduced to a group of students of fifth year of Biology. After seeing it, students completed, voluntarily and anonymously, a ten-item questionnaire, which considered the topics reflected in the film related with the educational objectives. Afterwards, a general discussion was carried out. They were asked to send a short essay with a personal thought about the activity.

Results. Fifty-two students (96.3\% of attendees) answered the questionnaire of evaluation. They considered that the movie had an outstanding interest to present the conflicts between science and social powers. They also agreed that such situations might still be present in the current world. Forty-nine students (90.7\%) sent the short essays. Students mainly wrote about the above-mentioned conflict, the barriers to knowledge, the discrimination against women and the need of respect for personal opinions.

Conclusions. Ágora may be a useful teaching tool to analyze and discuss the complex relationships among science, religion and politics. Even though it is set in the classical world, some of the issues posed in the movie may be still recognizable in our modern societies.

Key words. Heliocentrism. Politics. Popular movies. Religion. Science. Scientific method.

\section{Introducción}

La interpretación de la realidad siempre ha estado influida por las corrientes filosóficas imperantes en cada momento. En el mundo antiguo, la compren- sión de las enfermedades como fenómenos mágicos o religiosos no desapareció con medicina protocientífica griega [1]. En la misma dirección, el desarrollo de la ciencia basada en la observación y la experimentación no tardó en desafiar las creencias
Facultad de Ciencias de la Salud y de la Vida. Universitat Pompeu Fabra. Barcelona, España.

Correspondencia: Prof. Josep Eladi Baños Díez. Departamento de Ciencias Experimentales y de la Salud. Universitat Pompeu Fabra. Parc de Recerca Biomèdica de Barcelona. Dr. Aiguader, 88. E-08003 Barcelona.

Fax: +34933160901. E-mail: josepeladi.banos@upf.edu

Agradecimientos:

A los estudiantes de quinto curso de la licenciatura de Biología de la Universitat Pompeu Fabra (2010-2011), por su participación en esta experiencia y por contribuir a la evaluación con las respuestas al cuestionario que se les entregó.

Conflicto de intereses: No declarado.

Conflict of interests: None declared.

C 2012 Educación Médica 
Tabla I. Objetivos educativos de la sesión de 'Biocinema' en que se incluyó Ágora.

Analizar las dificultades de aceptar nuevas teorías científicas

Debatir el enfrentamiento entre principios religiosos, filosóficos y científicos

Establecer los efectos de las situaciones políticas sobre los hallazgos científicos

Comprender las interferencias entre ciencia, política y religión

Analizar cómo el fanatismo, la intolerancia y los intereses personales pueden influir en la aceptación de los datos cientíicicos

\section{Materiales y métodos}

Película

Ágora se estrenó en el año 2009 bajo la dirección de Alejandro Amenábar y con guión de él mismo y de Mateo Gil. Sus principales intérpretes son Rachel Weisz, Max Minghella y Oscar Isaac. El argumento se basa en lo acaecido a Hipatia, profesora de filosofía y astronomía en la Alejandría del siglo Iv, en un momento de turbulencias políticas en el Imperio Romano a las que no eran ajenas la conversión al cristianismo del emperador y los enfrentamientos entre cristianos, judíos y paganos en la ciudad alejandrina. En medio de todo ello, Hipatia sigue enseñando e intentando comprender el movimiento del universo a fin de conciliar la tradicional teoría geocéntrica de Ptolomeo con la observación del movimiento de los astros en el firmamento. Pero las luchas entre las religiones presentes en Alejandría desembocan en una confrontación política de la que Hipatia no queda ajena debido a la amistad con sus antiguos discípulos Orestes, prefecto romano de la ciudad, y Sinesio de Cirene, el obispo cristiano. La película recibió siete premios Goya en la edición de 2010, aunque todos menores a excepción del premio al mejor guión. La película es una recreación histórica de la Alejandría a finales del Imperio Romano y, aunque se toma algunas licencias históricas, incluye otros personajes de la época como el obispo Cirilo, que desempeña un papel importante en el devenir de todo lo que acontece en la película [13]. la organización del universo [3], la persecución de las teorías psicoanalíticas durante el nazismo [4] o, más recientemente, el rechazo a la teoría de la evolución darwiniana en amplios sectores de la sociedad estadounidense [5]. En los últimos años existen importantes debates, teóricamente científicos pero mucho más ideológicos, que conciernen a la medicina, como el aborto [6], la utilización de células madre [7], el uso de medios contraceptivos [8] o la prevención del sida [9], por citar sólo algunos. La comprensión de tales enfrentamientos es un elemento importante en la formación de los estudiantes universitarios. Sin embargo, pueden ser difíciles de transmitir a los estudiantes jóvenes, especialmente a los que han vivido siempre en una sociedad abierta y democrática.

Las películas comerciales han demostrado repetidamente su utilidad para debatir temas complejos en diversos ámbitos [10-12]. El presente artículo analiza la utilidad de Ágora para presentar las complejas relaciones entre religión, política y ciencia y debatir su persistencia en el mundo contemporáneo.

\section{Actividad}

La película formó parte de la actividad docente denominada 'Biocinema' [14], cursada por los estudiantes de quinto curso de Biología de la Universitat Pompeu Fabra a lo largo del primer trimestre del curso 2010-2011. La actividad se desarrolló como sigue. En primer lugar, uno de los profesores responsables (J.A.) presentó la película y a continuación se procedió a su proyección para todo el grupo. Después se pidió a los estudiantes que respondieran de forma anónima y voluntaria un cuestionario (véase la sección siguiente) y se procedió después al debate abierto de la película, moderado por el mismo profesor durante un período aproximado de una hora. Después se dio por finalizada la actividad. Como evaluación se solicitó a los estudiantes que lo desearan que prepararan un informe personal en forma de un ensayo corto (1-2 páginas) con una reflexión sobre la actividad. Este texto se remitía a los tres profesores responsables 
Tabla II. Cuestionario de evaluación de la actividad.

\begin{tabular}{|c|c|}
\hline Preguntas & Posibles respuestas \\
\hline 1. ¿Habías visto previamente la película? & Sí / No \\
\hline 2. ¿Te ha interesado la película? & 1: nada; 10: mucho \\
\hline $\begin{array}{l}\text { 3. ¿Crees que plantea de forma adecuada los conflictos entre } \\
\text { el poder político y el religioso con el pensamiento científico }\end{array}$ & 1: nada; 10: mucho \\
\hline 4. ¿Crees que en el mundo actual se plantean también estos conflictos? & 1: nada; 10: mucho \\
\hline $\begin{array}{l}\text { 5. ¿Estás de acuerdo con la afirmación de que los científicos han } \\
\text { de evitar las confrontaciones con los poderes políticos o religiosos? }\end{array}$ & 1: nada; 10: mucho \\
\hline 6. ¿Cuál es la razón por la que Hipatia fue asesinada? & $\begin{array}{l}\text { 1: compromiso político; 2: falta de compromiso religioso; } \\
\text { 3: condición femenina; 4: ideas científicas; 5: otras (especifíquese) }\end{array}$ \\
\hline $\begin{array}{l}\text { 7. ¿Crees que las creencias religiosas y filosóficas } \\
\text { influían en la teoría geocéntrica del universo? }\end{array}$ & 1: nada; 10: mucho \\
\hline $\begin{array}{l}\text { 8. ¿Crees que, en la actualidad, las creencias y los } \\
\text { sentimientos personales pueden influir en la ciencia? }\end{array}$ & 1: nada; 10: mucho \\
\hline 9. ¿Por qué era difícil refutar la teoría geocéntrica de Ptolomeo? & $\begin{array}{l}\text { 1: razones religiosas; } 2 \text { : paradigmas establecidos; } 3 \text { : dificultades } \\
\text { experimentales; } 4 \text { : concepciones filosóficas; } 5 \text { : otras (especifíquese) }\end{array}$ \\
\hline 10. ¿Cuál es, en tu opinión, el tema central de la película? & $\begin{array}{l}\text { 1: la intolerancia; 2: el fanatismo; 3: el amor; 4: la ciencia; } \\
\text { 5: otras (especifíquese) }\end{array}$ \\
\hline
\end{tabular}

11. ¿Quieres añadir algún comentario?

de los itinerarios profesionales (J.A., F.B., M.S.) para su evaluación.

\section{Evaluación}

Para establecer si la actividad permite cumplir los objetivos educativos preestablecidos (Tabla I) se utilizaron dos elementos. El primero fue un cuestionario de diez preguntas mediante el cual se evaluaban diversos aspectos vinculados a la actividad: aspectos generales (visionado previo de la película, interés para el estudiante), aspectos específicos de la película (planteamiento de los problemas a debate, razones que llevan al asesinato de Hipatia, conflictos de ciencia con religión, confrontación entre teoría heliocéntrica y geocéntrica, tema central de la película) y opinión sobre la existencia actual del problema (problemas similares en el mundo contemporáneo, conocimiento de cuándo se aceptó la teoría heliocéntrica). Las preguntas del cuestionario se recogen en la tabla II. El segundo elemento de evaluación fue el informe personal descrito en la sección anterior.
Los resultados de la evaluación del cuestionario se expresan como media \pm desviación estándar cuando los resultados fueron numéricos (preguntas 2, 3, 4, 5, 7 y 8), y como distribución de frecuencias en el resto (preguntas 1, 6, 9 y 10). El apartado de 'Resultados' incluye una evaluación cualitativa de los informes personales realizados por los estudiantes.

\section{Resultados}

\section{Cuestionario de evaluación}

De los 54 estudiantes que asistieron a la sesión, 52 (96,3\%) respondieron el cuestionario. De ellos, 35 $(67,3 \%)$ afirmaron no haber visto la película antes de la sesión de 'Biocinema'. En el apartado de comentarios, varios de ellos incluyeron reflexiones positivas ('es adecuada para el ciclo', 'muy buena, pensaba que era peor según lo que había oído', 'me ha gustado mucho, pues es una forma diferente de debatir sobre la ciencia'), aunque también se recogió alguna crítica ('es una película demasiado lenta'). 
Figura. Porcentaje de respuestas a las preguntas no numéricas. Cada pregunta podía responderse con más de una opción posible. En abscisas se representan las tres preguntas con sus cinco posibles opciones de respuesta (entre paréntesis, el total de respuestas). Pregunta 6: '¿cuál es la razón por la que Hipatia fue asesinada?'. 1: compromiso político; 2: falta de compromiso religioso; 3: condición femenina; 4: ideas científicas; 5: otras. Pregunta 9: '¿por qué era difícil refutar la teoría geocéntrica de Ptolomeo?'. 1: razones religiosas; 2: paradigmas establecidos; 3 : dificultades experimentales; 4: concepciones filosóficas; 5: otras. Pregunta 10: '¿cuál es, en tu opinión, el tema central de la película?' . 1: la intolerancia; 2: el fanatismo; 3: el amor; 4: la ciencia; 5: otras.

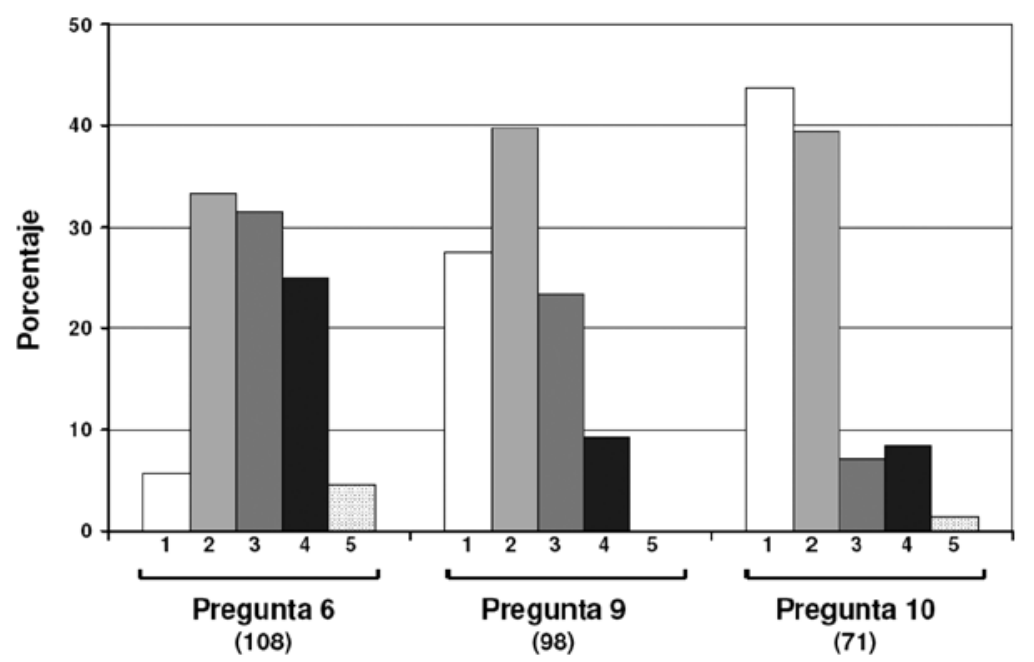

La tabla III recoge los valores que los estudiantes concedieron a las preguntas a las que se solicitaba un valor numérico. Como puede observarse, la mayoría de las respuestas se agruparon en el intervalo de 6 a 10, excepto la pregunta sobre la necesidad de que los científicos eviten los conflictos con los poderes políticos y religiosos, donde las respuestas se dividieron de forma casi idéntica entre el intervalo superior (6-10) y el inferior (1-5), con un valor medio de 5,8. De las respuestas aportadas se deduce que los estudiantes consideraron la película de un interés notable (media de 7,9), que plantea de forma adecuada los conflictos entre el poder político y religioso y el pensamiento científico $(8,1)$, que tales conflictos aún se dan en la actualidad $(7,5)$, que las creencias religiosas y filosóficas contribuyeron al mantenimiento de la teoría geocéntrica (9) y que las impresiones subjetivas (creencias y sentimientos) todavía pueden influir en la actualidad en la ciencia $(6,3)$.

En las preguntas en que se dieron varias opciones de respuesta y donde podían incluso elegir más de una, las respuestas fueron más variables (Figura). Por ejemplo, en la que se solicitaba cuál era la razón del asesinato de Hipatia, se obtuvieron 108 res- puestas (2,1 por estudiante). En este caso, las más frecuentes fueron la falta de compromiso religioso $(n=36 ; 33,3 \%)$, la condición femenina $(n=34$; $31,5 \%)$ y las ideas científicas $(n=27 ; 25 \%)$. En las respuestas abiertas, se señaló su compromiso con la ciencia y su fe en la filosofía, el pensamiento de nuevas ideas y el pensamiento crítico. Cuando se solicitó por qué era difícil refutar la teoría geocéntrica de Ptolomeo, de nuevo las respuestas estuvieron divididas. Se obtuvieron 98 opciones (1,9 respuestas por estudiante) y las más frecuentes fueron los paradigmas establecidos $(n=39 ; 39,8 \%)$, seguido de las razones religiosas $(n=27 ; 27,5 \%)$ y las dificultades experimentales $(n=23 ; 23,5 \%)$. En el caso del tema central de la película, las respuestas obtenidas fueron 71 (1,4 por estudiante), que se agruparon básicamente en dos: la intolerancia $(n=31 ; 41,3 \%)$ y el fanatismo $(n=28 ; 37,3 \%)$. Entre las respuestas abiertas a esta pregunta aparecieron las opciones de los conflictos religiosos, la ignorancia y la búsqueda del poder a cualquier precio.

\section{Informes personales}

De los estudiantes que asistieron a la sesión, 49 (90,7\%) enviaron los informes personales. Sobre el interés de la película, las palabras de un alumno muestran cómo el escepticismo inicial cambió a lo largo de la actividad: 'aunque en un principio no creía que esta película se ciñera al objetivo que se perseguía con esta actividad, creo que me ha hecho reflexionar mucho y me ha obligado a plantearme preguntas que creía que tenían una más fácil respuesta.'

Los principales temas que consideraron en los informes personales se resumen en la tabla IV. Como puede observarse, seis centraron su atención: los conflictos entre poderes políticos y religiosos con el conocimiento científico, las barreras y los retos del conocimiento en general y el científico en particular, la discriminación de la mujer en la sociedad y en la ciencia, el crecimiento y la expansión del cristianismo, y el respeto por el pensamiento individual.

Los estudiantes se interesaron por destacar hasta qué punto el pensamiento individual representa una amenaza para el poder político y el eclesiástico, incluso en el momento actual, en el que se entrevé un recelo cuando se cuestiona la validez del sistema económico, la organización política o la influencia de la Iglesia. También despertó numerosos comentarios el papel de la mujer en la sociedad y cómo Hipatia representaba una situación que aún hoy es reconocible. En este sentido se destacaba el escaso protagonismo que tradicionalmente la mujer ha te- 
Tabla III. Valores que concedieron los estudiantes a las preguntas que tenían una respuesta numérica (1: nada; 10: mucho)

\begin{tabular}{lccc}
\hline & $n \leq$ valor 5 & Rango & $\begin{array}{c}\text { Media } \pm \\
\text { desviación estándar }\end{array}$ \\
\hline Interés de la película & $2(3,8 \%)$ & $4-10$ & $7,96 \pm 1,66$ \\
\hline Planteamiento de conflictos entre poder político/religioso y pensamiento científico & $2(3,8 \%)$ & $1-10$ & $8,10 \pm 1,50$ \\
\hline Actualidad de estos conflictos & $6(11,5 \%)$ & $3-10$ & $7,53 \pm 1,62$ \\
\hline Evitación de confrontación con poder político/religioso & $27(51,9 \%)$ & $1-10$ & $5,73 \pm 3,25$ \\
\hline Creencias religiosas/filosóficas en la teoría geocéntrica & $3(5,8 \%)$ & $3-10$ & $9,00 \pm 1,55$ \\
\hline En la actualidad, las creencias y sentimientos pueden influir en la ciencia & $15(29 \%)$ & $1-10$ & $6,32 \pm 2,49$ \\
\hline
\end{tabular}

nido en el mundo científico y la esperanza que esta situación pueda cambiar en el futuro inmediato con su incorporación a la universidad y al mundo científico, aunque se manifestaba un cierto pesimismo en que la igualdad pueda alcanzarse a corto plazo. Sin embargo, los estudiantes no demostraron poseer un conocimiento sólido sobre las causas histórico-sociales de estas diferencias. Asimismo, se debatió el conflicto que enfrenta la religión, promotora de una visión e interpretación del hombre y el universo basado en dogmas, con el conocimiento científico, basado en la proposición y refutación de hipótesis mediante la observación y la experimentación. A este respecto, se comentó que el recelo, e incluso el rechazo, del conocimiento científico por parte de las autoridades religiosas a lo largo de la historia reciente de Occidente representa una manifestación del temor a la pérdida del poder sociopolítico, ya que la religión y el poder político han estado muy ligados en nuestra sociedad.

Finalmente, se podría resumir esta segunda parte de la evaluación en que los puntos de debate han sido muchos y variados en los informes de los estudiantes. El más importante sería el análisis de cómo la religión, la filosofía y el poder pueden llegar a constituir una verdadera coalición que se enfrenta con frecuencia con el conocimiento científico. Y este debate, visto desde la perspectiva de una mujer y la discriminación que sufre. Según los estudiantes, no deja de sorprender que la comparación entre lo que ocurría en el siglo IV y en el siglo XXI no depara tantas diferencias como cabría esperar. En palabras de una estudiante, 'creo que esta película plantea una época donde los conflictos religiosos marcaban a la sociedad. Es importante analizar sus consecuencias porque es posible que, por culpa de fanáticos religiosos o políticos, en la actualidad se repita en cierta medida.'

\section{Discusión}

Los conflictos entre los científicos y los poseedores en cada momento del poder político (eclesiásticos y laicos como reyes o emperadores) no han sido infrecuentes en la historia del mundo occidental. Recuérdense como ejemplo los casos dramáticos entre la Iglesia romana y Galileo [15]. Parece que René Descartes no publicó parte de su obra más fisiológica por el miedo a ser perseguido por la Inquisición y hay quien defiende que su teoría del dualismo mente-cuerpo era, más que una disquisición mecanicista, una forma de evitar entrar en conflicto con la Iglesia de la época sobre la posibilidad de estudiar la mente, que aquella asociaba con el alma [16]. En tiempos recientes, diversas polémicas han mostrado que tal conflicto se encuentra lejos de desaparecer [5-9], por lo que la posibilidad de que la actividad científica pueda ser influida por creencias religiosas o morales sigue siendo un tema de actualidad. Además, a lo largo de la historia, la situación se complica por la influencia de otros factores, como la consideración del papel de la mujer o la negación apriorística de los descubrimientos científicos cuando contradice el dogma religioso. No es arriesgado afirmar que la conjunción de todos estos factores en un momento determinado puede llevar a situaciones de violencia extrema que pueden segar, aun hoy, la vida de algunos de sus protagonistas [17].

La formación de los universitarios en ciencias, básicas o aplicadas, no puede limitarse a una transmisión de conocimientos y a la adquisición de unas 
Tabla IV. Principales temas considerados en los informes de los estudiantes con algunos ejemplos de cada grupo.

Conflictos entre los poderes políticos y religiosos con el conocimiento científico

Filosofía y religión como elementos de oposición al conocimiento

y cómo han contribuido a limitar el progreso científico

Rechazo de las teorías científicas (heliocentrismo) por motivos filosóficos o religiosos

Influencia recíproca entre creencias religiosas y pensamiento científico

Problema de los dogmatismos, fanatismos y fundamentalismos

Diferencias entre la concepción religiosa del mundo (basada en dogmas)

y la científica (basada en hipótesis, observación y experimentación)

Barreras y retos del conocimiento en general y el científico en particular

Necesidad de la crítica con las ideas establecidas y el inconformismo científico como motor de progreso

Fomento de la ignorancia como mecanismo de perpetuación de poder

Problemática de la ciencia, representada por la astronomía, pero aplicable a cualquier disciplina científica

Percepción del pensamiento científico como una amenaza al poder

Limitación de la investigación científica por la intervención política

(áreas de interés, líneas preferentes, financiación)

Influencia de las creencias individuales (apriorismos) en las observaciones científicas

Existencia de temas 'tabú' en las diferentes sociedades

Discriminación de la mujer en la sociedad y en la ciencia

Papel de la mujer en la sociedad, representado por el enfrentamiento de Hipatia

Razones del menosprecio y la marginación de la mujer

Papel de la mujer frente al hombre a lo largo de la historia

Distinta consideración de hombre y, mejor, en las religiones

Presencia en la sociedad actual de algunos de los conflictos planteados por la película

Ejemplos actuales de la influencia de los poderes religiosos y políticos en la ciencia

Ejemplos de discriminación por razón de sexo en la ciencia y en la sociedad

Diferencias de la situación según los países

Crecimiento y expansión del cristianismo

Enfrentamientos entre cristianos, paganos y judíos

Comparación del conflicto de la película con las guerras religiosas

Respeto por el pensamiento individual

Recelo de los poderosos frente a quienes cuestionan el poder económico, político, religioso o científico

Individualismo como amenaza de los poderes establecidos

Necesidad del respeto de las opiniones minoritarias

habilidades experimentales. Cualquier profesión tiene compromisos éticos que deben explorarse de la forma más factible durante el devenir de los estu- diantes por la universidad. Las actividades descritas bajo el nombre de 'Biocinema' pretenden que los estudiantes sean capaces de analizar los compromisos morales que pueden implicar sus actividades profesionales una vez graduados [14]. Algunos de estos compromisos son difíciles de abordar y el empleo de películas comerciales facilita el inicio del análisis y del debate a través de recursos cinematográficos. Sin embargo, obtener una película de suficiente interés para alcanzar los objetivos previstos no es siempre tarea fácil. Por esta razón, antes de implementarla en un programa regular, resulta deseable analizar cuidadosamente la participación, la opinión y las respuestas de los estudiantes en experiencias piloto como la que aquí se presenta.

En el presente artículo se exponen los resultados de la evaluación realizada con el primer grupo de estudiantes que participaron en la sesión de 'Biocinema' con la película Ágora. Las razones para utilizarla fueron consecuencia de la publicación de diversos artículos que destacaban su interés para explicar algunos aspectos de cómo funcionan los componentes sociales de la ciencia [18-21] e incluso la concesión de un premio en un festival de cine científico [22]. En nuestra actividad, Ágora pretendía presentar a los estudiantes la sutil $-o$ a veces obvia- influencia de factores sociales paracientíficos (filosóficos, políticos, religiosos) sobre la interpretación de las teorías especulativas o los datos científicos. Los resultados obtenidos, aunque con una pequeña muestra, tienen un interés notable y se comentan brevemente a continuación.

En primer lugar, llama la atención el hecho de que una película reciente como Ágora sólo haya sido vista previamente por un tercio de los participantes en el estudio. Ello aconseja no prejuzgar contra películas de estreno reciente porque es posible que una fracción importante de los estudiantes no la hayan visto. En cualquier caso, el interés de la película se consideró elevado, con una puntuación media de casi 8 , y con la gran mayoría otorgando valores superiores a 5 en una escala de 1 a 10 . Obviamente, este es un aspecto secundario en la actividad, donde lo que se desea es alcanzar los objetivos educativos previstos, pero es un buen indicador del seguimiento de la película.

En opinión de los estudiantes, la película plantea de forma adecuada los conflictos entre el poder político y religioso con el pensamiento científico, con lo que uno de los principales objetivos de la actividad podría cumplirse con esta percepción. Los estudiantes también consideraron que tales conflictos se reproducen en el mundo actual, lo que permitió un debate sobre algunos ejemplos, así como de la 
dificultad de tratarlos. La aceptación de que en la actualidad las creencias y sentimientos personales también pueden influir en la ciencia presentó una menor unanimidad, con una tendencia a aceptar esta proposición. También hubo una mayor división entre aquellos que pensaban que debían evitarse tales confrontaciones y los partidarios de mantener los principios científicos aunque ello suponga un enfrentamiento claro con la autoridad política y religiosa. Esto sugiere un notable grado de sinceridad en los participantes que no se alinearon hacia una posición más 'idealista' de rebeldía frente a los poderes establecidos, aunque podría leerse también como una postura de claudicación frente a éstos. Existió en cambio una notable unanimidad sobre el efecto de las creencias religiosas y filosóficas en la aceptación de la teoría geocéntrica. Las razones aportadas para comprender la dificultad de refutarla se basan, en primer lugar, en los obstáculos en superar los paradigmas establecidos, y en menor lugar, en las razones religiosas y las dificultades experimentales. Estos resultados muestran la comprensión hacia cómo lo aceptado como verdad inamovible puede tener grandes dificultades para ser refutado.

Uno de los apartados del cuestionario interrogaba sobre cuál creían que había sido la razón que llevó al asesinato de Hipatia. En esta cuestión hubo una gran división de opiniones, especialmente las que atribuían su muerte a la falta de compromiso con la nueva religión imperante y a su condición de mujer, dejando en tercer lugar las ideas científicas. En nuestra opinión, esta es una opinión bastante acertada porque Hipatia reúne dos cualidades que la hacen especialmente vulnerable en la situación de extremo fanatismo que refleja la película: la falta de vinculación con el nuevo orden religioso y su condición de mujer influyente, a la que se desacredita claramente durante una lectura realizada en una ceremonia religiosa que se muestra en la película. Vinculada a esta pregunta se encuentra la última cuestión que incluía el cuestionario de evaluación, la referida a cuál era el tema central de la película. La mayoría de los participantes señalaron la intolerancia y el fanatismo como los principales elementos del argumento, lo que permite contribuir al establecimiento de cómo tales conductas pueden influir en la aceptación del hecho científico e incluso a refutarlo.
En conclusión, los resultados de este estudio sugieren que Ágora puede contribuir a la comprensión de las difíciles relaciones entre ciencia, política y religión en aquellos aspectos en que los datos experimentales cuestionan creencias establecidas o dogmas que constituyen aspectos nucleares de las dos últimas. Quizá, como destacaba uno de los estudiantes, una frase de Hipatia resume todo el conflicto que narra la película: 'tú no cuestionas lo que crees, yo debo'.

\section{Bibliografía}

1. López-Piñero JM. La medicina en la historia. Madrid: La Esfera de los Libros; 2002.

2. Dampier WC. Historia de la ciencia y sus relaciones con la filosofía y la religión. Madrid: Tecnos; 1986.

3. Ordóñez J, Navarro V, Sánchez-Ron JM. Historia de la ciencia. Madrid: Espasa-Calpe; 2007.

4. Frosh S. Psychoanalysis, Nazism and 'Jewish science' Int J Psychoanal 2003; 84 (Pt 5): 1315-32.

5. De Vries A. The enigma of Darwin. Clio Med 1984; 19: 136-55.

6. Castle MA. Abortion in the United States' bible belt: organizing for power and empowerment. Reprod Health 2011; 8: 1.

7. Tauer CA. International policy failures: cloning and stem-cell research. Lancet 2004; 364: 209-14.

8. Mishtal J, Dannefer R. Reconciling religious identity and reproductive practices: the Church and contraception in Poland. Eur J Contracept Reprod Health Care 2010; 14: 232-42.

9. Condoms and the Vatican [editorial]. Lancet 2006; 367: 1550.

10. Bowman J. Bioethics at the movies. New Atlantis 2005; 8: 93-100.

11. Lumlertgul N, Kijpaisalratana N, Pityaratstian N, Wangsaturaka D. Cinemeducation: a pilot student project using movies to help students learn medical professionalism. Med Teach 2009; 31: e327-32.

12. Datta V. Madness and the movies: an undergraduate module for medical students. Int Rev Psychiatry 2009; 21: 267-8.

13. Ágora (portal de la película). URL: http://www.agoralapelicula. com. [14.02.2011].

14. Baños JE, Aramburu F, Sentí M. Biocinema: la experiencia de emplear películas comerciales con estudiantes de Biología. Rev Med Cine 2005; 1: 42-6.

15. Gribbin J. Historia de la ciencia. Barcelona: Crítica; 2003.

16. Duncan G. Mind-body dualism and the biopsychosocial model of pain: what did Descartes really say? J Med Philos 2000; 25: 367-77.

17. Alandete D. George Tiller, médico abortista asesinado en Estados Unidos. El País, 6 de junio de 2009.

18. Firestein S. Better reception in Europe? Science 2010; 328: 1482-3.

19. Knight J. Hollywood or bust. Nature 2004; 430: 720-2.

20. Scott AO. Love amid the togas and the intolerant. The New York Times, 28 de mayo de 2010.

21. Rohter L. Science vs. Zealots, 1,500 years ago. The New York Times, 23 de mayo de 2010.

22. Hamptons Online. URL: http://www.hamptons.com/ The-Arts/Hamptons-International-Film-Festival/9133/ HIFF-And-Alfred-P.-Sloan-Foundation-Celebrate-Ten.html. [14.02.2011]. 